healthcare professionls should have greater awareness. This has been supported by the recent RCPCH position statement calling for practical sign-posting. ${ }^{7}$

\section{G78 A SIMPLE SCREENING METHOD FOR THE MEASUREMENT OF LYSOSOMAL ACID LIPASE USING DRIED BLOOD SPOTS}

doi:10.1136/archdischild-2013-304107.090

J Hamilton, I Jones, R Srivastava, P Galloway. Biochemistry Department, Yorkhill Hospital, Glasgow, UK

Background Deficiency of the enzyme lysosomal acid lipase (LAL) presents in infancy with hepatosplenomegaly, adrenal calcification and failure to thrive - Wolman Disease (WD). Cholesterol Ester Storage Disease (CESD) is the more common and milder form and presents later with hepatomegaly and hyperlipidaemia. Until recently there was no recognised method for the measurement of LAL in dried blood spots (DBS).

Aim To develop a simple method for the measurement of lysosomal acid lipase in DBS and validate the technique using samples from patients with CESD.

Methods LAL was extracted from DBS with water and incubated with $4 \mathrm{mU}$-palmitate in acetate buffer and cardiolipin as an activator of LAL. Lalistat 2 is used as an inhibitor of LAL and activity is estimated by measuring total lipase activity and activity in the presence of Lalistat 2. LAL was determined by subtracting activity measured in the inhibited reaction from that in the uninhibited reaction. The assay was performed using 96-well plates and read at $355 \mathrm{~nm} / 460 \mathrm{~nm}$.

Results LAL activity in normal controls was $0.37-2.30 \mathrm{nmol} /$ punch/hr. Samples from CESD patients and WD have significantly reduced LAL activity : $<0.04 \mathrm{nmol} / \mathrm{punch} / \mathrm{hr}(\mathrm{n}=32)$. Activity in samples from obligate carriers is in the range $0.15-0.40 \mathrm{nmol} /$ punch/hr $(\mathrm{n}=15)$.

Conclusions Until now, methods for the measurement of LAL required the use of leucocytes and fibroblasts.

These are difficult samples to prepare and methods for the measurement of LAL using these sample types are time consuming and expensive. Measurement of LAL in DBS is made difficult by the presence of other lipases in whole blood. Lalistat 2 is a specific inhibitor of LAL which allows the reliable determination of LAL in DBS Results show the method differentiates clearly between normal controls, carriers and affected cases.

The DBS sample is simple to prepare and transport. By performing the assay using 96-well plates, samples can be processed efficiently in batches, previously not possible. Therefore it is now practical to screen for WD and CESD in patients with a lower index of suspicion of the disorder.

\section{G79 NUTRITIONAL STATUS OF INFANTS WITH CONGENITAL HEART DISEASE}

doi:10.1136/archdischild-2013-304107.091

P Siddhi, W Kelsall, F Young, E Johnson. Neonates and Paediatrics, Cambridge University Hospital, Cambridge, UK

Aims To assess the nutritional status of infants with congenital heart disease (CHD) who were managed jointly by the medical and dietetic teams and required either high calorie feeds or nasgastric feeding to optimise their growth.

Methods Infants with CHD requiring dietetic input between 2008-2011 were reviewed. Patients were identified from paediatric cardiac and dietitians databases. A retrospective case note and electronic clinic letter review was performed.

Results Thirty nine infants were studied: 15(39\%) with cyanotic CHD and 24(61\%) acyanotic CHD. In 12 (29\%) cases CHD was part of a syndrome. $25(64 \%)$ were treated surgically, $8(21 \%)$ required transcatheter procedures and $6(15 \%)$ were managed medically. Surgery was performed in $9(27 \%)$ within 1-month of their birth and $24(73 \%)$ within the first-year.10 (67\%) of the cyanotic infants had their corrective surgeries in the first 6 months. All 6 $(15 \%)$ managed medically were among the acyanotic infants, 19 $(76 \%)$ of the acyanotic infants were on diuretics and $10(40 \%)$ were operated in the later half of the year. There were $4(10 \%)$ deaths in this cohort.

The birthweight was less than the 2 nd centile in $12 \%$ of infants and overall $67 \%$ had a birthweight less than 50 th centile. In the preoperative period, 14(41\%) were nasogastric fed (NG). The majority of infants were on mixed feeds, $50 \%$ receiving some breast milk and $65 \%$ receiving Infatrini to maximise the weight gain. In the postoperative period, $10(30 \%)$ needed nasogastric feeds and 3(8\%) with complex gut anomalies needed gastrostomy feeding tubes. The NG tubes were in place post-operatively for a median period of 2 months. At the end of 1 year, there was improvement in weight gain in $8(53 \%)$ of the cyanotic infants and $9(38 \%)$ of the acyanotic infants.

Conclusion Challenges persist in optimising the nutritional needs especially in children with CHD particularly those on prolonged diuretics. Collaboration with the dieticians is essential for optimising their growth prior to and after surgery.

\section{G80 PRESENCE AND PROGRESSION OF IDIOPATHIC DILATED CARDIOMYOPATHY}

doi:10.1136/archdischild-2013-304107.092

AN Kanani, A Lowdon, A Adigun, A Chikramane. Department of Cardiology, Birmingham Childrens Hospital, Birmingham, UK

Aim Idiopathic Dilated cardiomyopathy (iDCM) is an uncommon presentation in children and young people but with significant and quite often poor prognosis. We aim to look at the presence and progression of iDCM.

Material and Methods 12 year retrospective analysis of data in a single centre; all patients with the term "DCM" were reviewed from the cardiac database (36 patient). A diagnosis was made on clinical and echocardiographic criteria. We excluded any patient where a cause was identified, analysed sequential echocardiograms and ECGs, and evaluated their progression and outcome.

Results There were 24 patients with iDCM; 15 boys and 9 girls. Presentation age range was Birth - 16 years years (mean: 5 years 8 months). Children/adolescents were followed up for an average of 80-months (range: 6 - 143 months). We measured LV dimensions and ventricular function and " $z$ " scores calculated to track growth along with valvar lesions.

22 were on medication; b-blockers in 16 , and 21 had an ACE inhibitor/ARB. 6 had additional medication in the form of Spironolactone and (12) diuretics. Anti-platelet (12)) use was also studied. 3 underwent transplant and 2 were referred for a transplant and adult cardiology review. 3 patients who had CRT. Ventricular function was tracked to the point of the last review. Clinical improvement was noted on NYHA criteria.

We had an adverse outcome (death/transplant) in $17 \%$, death in 4 with 1 going on to have a transplant within this period. Of the survivors, we tracked their NYHA grade at the last review - (I, through IV). The fractional shortening was $(7-28 \%)$ at presentation, (mean 18\%), and $10-34 \%$ at discharge (mean $25 \%$ ).

Of those who died, the mean FS was $17 \%$, and $28 \%$ in the survivors. LV diastolic dimension (LVDd, z scored) similarly was noted at presentation and at last review.

Conclusion We found that there was an adverse outcome in 17\%. Actuarial survival was $100 \%$ at 1 year and $8.3 \%$ at 5 years with the best outcomes noted in younger age groups and in those where there was an improvement in systolic function. 